\title{
Pathological concepts in acute coronary thrombosis: relevance to treatment
}

\author{
William F M Fulton
}

\section{Coronary thrombosis and myocardial infarction}

It is a little over a decade since the acute management of myocardial infarction underwent a revolution with direct intervention on the coronary occlusive lesion. This approach had validity only if the coronary occlusion caused infarction. The evidence generated by coronary angiography soon after the onset of pain, the frequent restoration of coronary blood flow by therapeutic thrombolysis, and the apparent benefit to the threatened myocardium soon dispelled any reasonable clinical doubt that thrombotic occlusion was the prime cause of myocardial infarction. It had not always been so. ${ }^{1}$

For more than half a century pathologists had been comfortable with the orthodox concept that coronary thrombosis was the cause of myocardial infarction. In the 1970's, however, some pathologists proposed the maverick concept that coronary thrombosis was inconstant and was a secondary consequence of infarction. ${ }^{2}$

The validity of the orthodox concept was re-examined by several workers. A prevalence of thrombotic occlusion in regional myocardial infarction of some $90-97 \%$ was revealed. ${ }^{3-6}$ Post-mortem evidence being of necessity retrospective, uncertainties about the time relation between occlusion and infarction persisted. In 1974 I started a prospective clinico-pathological necropsy study that used radiofibrinogen given in life as a marker of the timing of thrombus formation. A $100 \%$ incidence of acute coronary occlusion was confirmed in acute fatal myocardial infarction, all but one lesion being thrombotic. Also certain aspects of the study, in particular retrospective anatomical reconstruction of the underlying plaque and the demonstration of the different ages of thrombus, have contributed to the understanding of the clinical features of acute ischaemic syndromes.

A substantially lower incidence and even the absence of coronary thrombosis has usually been reported in diffuse subendocardial infarction. ${ }^{15-8}$ It is important to distinguish this entity from regional infarction. ${ }^{9}$ Diffuse subendocardial damage occurs in the presence of widespread severe obstructive coronary disease. Critically severe ischaemia is equitably distributed by greatly enlarged anastomoses, which are particularly extensive in the subendocardial plexus. ${ }^{7-10}$ The putative final insult is usually extracoronary-for example, tachycardia, hypoxia, or heart failure-but it can be a fresh thrombotic occlusion.

\section{Prospective clinico-pathological trial 1974-1979}

${ }^{125} \mathrm{I}$-labelled fibrinogen $(100 \mu \mathrm{Ci})$ was administered intravenously soon after admission to the coronary care unit. In fatal cases, postmortem examination included stereoarteriography and serial mounting of $2 \mathrm{~mm}$ thick transverse sections of coronary arteries. ${ }^{8}$ Autoradiography of the mounted sections identified the distribution of radiolabelled fibrin in thrombus formed after the introduction of the isotope label (fig 1). The relative intensity of radioactivity was graded on a scale of 0-256. Thrombus already formed before the administration of labelled fibrinogen was of zero or very low activity. Stereoarteriograms and serial $2 \mathrm{~mm}$ thick transverse slices permitted reconstruction of the microanatomy of obstructive lesions.

The primary objective of this study was to define as far as possible the relation between coronary occlusion and acute myocardial infarction. This was achieved. Fifty seven cases of acute myocardial infarction were given radioactive fibrinogen soon after admission. At necropsy 56 had thrombotic occlusion and one had occlusion caused by atheromatous debris of the artery subtending the infarct. The opportunity was also taken to explore or re-affirm many relevant aspects of the microanatomy and morphogenesis of coronary occlusive lesions.

In 47 of the 56 cases a central portion of thrombus was radionegative: that is it had already formed before radiolabelled fibrinogen was given. Figure 2 shows the typical distribution of radiopositive and radionegative thrombus found in most cases. The interval between clinical onset to administration of radiolabelled fibrinogen was $<3$ hours in six, $<4.5$ hours in $12,<6$ hours in 16 , and $<12$ hours in 31 cases, cumulative. Radiolabelled thrombus could be found both proximal and distal to the unlabelled central nidus, showing that thrombus continues to propagate after the inception of infarction. ${ }^{11}$ In the remaining nine cases the central portion of the thrombus was radiopositive. In seven of these the infarct clearly evolved from unstable angina or developed further infarction after radiofibrinogen was given. 


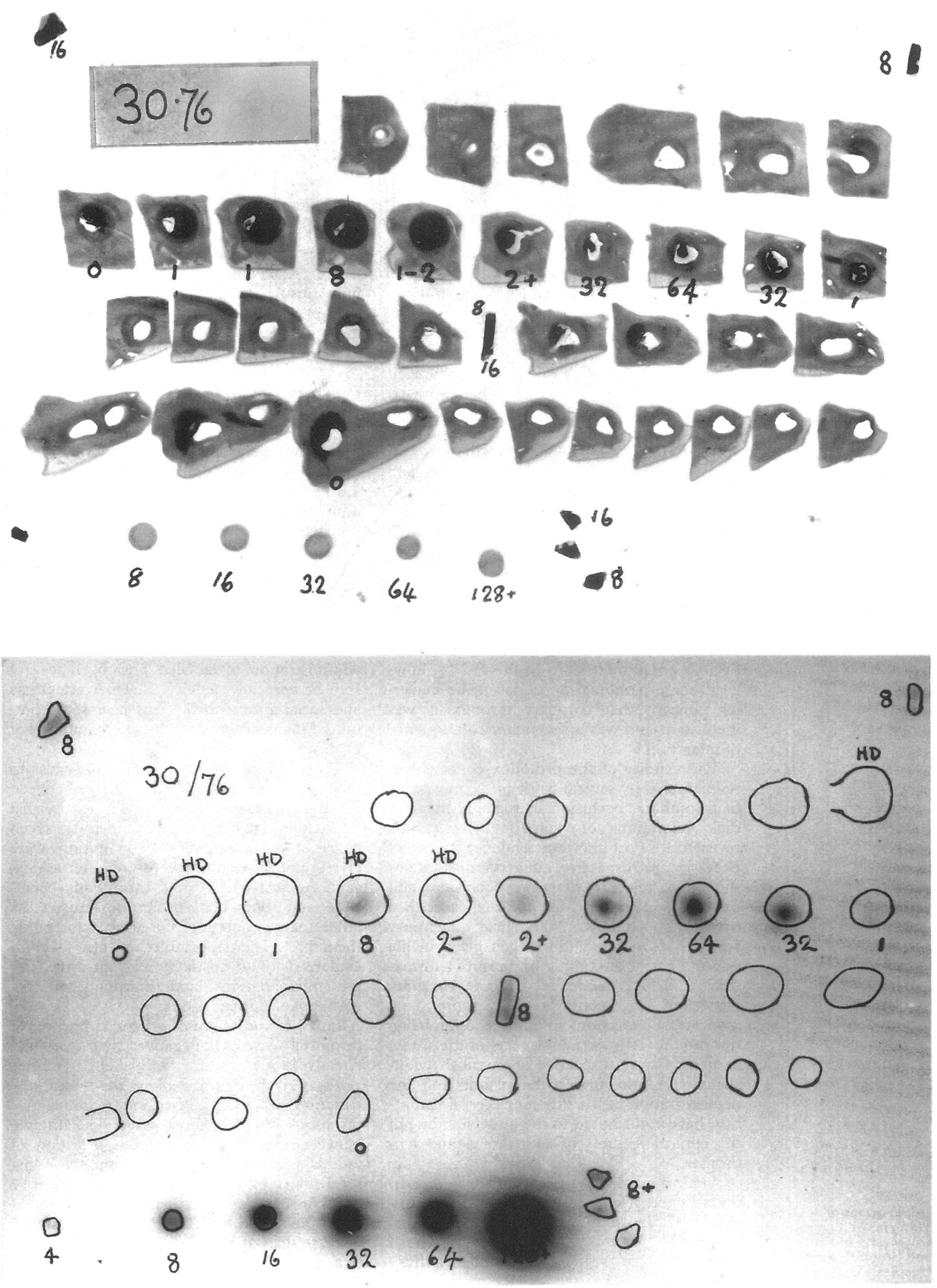

Figure 1 (Upper) Serially mounted $2 \mathrm{~mm}$ thick transverse sections of a right coronary artery. (Lower) Autoradiograph prepared from $A$ by near-contact exposure for 7 days with superimposed transfer drawing of coronary artery outlines with radioactive standards and portions of cardiac chamber thrombus as markers for registration. The complete occlusion at the fifth section in row 2 is by primary thrombus of very low radioactivity (grade 1-2, see text). Propagated thrombus of higher radioactivity is present proximally and distally-for example, section 8 second row grade 64. (Case history: Unstable angina 3 weeks, onset of acute myocardial infarction to radiofibrinogen administration 5.5 hours; administration of radiofibrinogen to death 35 hours). HD, haemorrhagic dissection. $(\times 1 \cdot 3)$ 
Figure 2 Diagram representing the distribution of radiolabelled fibrinogen in typical coronary thrombi. The central, putatively

"primary", thrombus has 0 activity and the

"secondary" propagated thrombus has high radioactivity. Low grades of radioactivity are seen at margins of central

thrombus. Grade 0-4 relative radioactivity classed as "cool", in keeping with thrombus formation before radiofibrinogen was introduced. Relative radioactivity of whole blood clot is grade 16 (8-32).

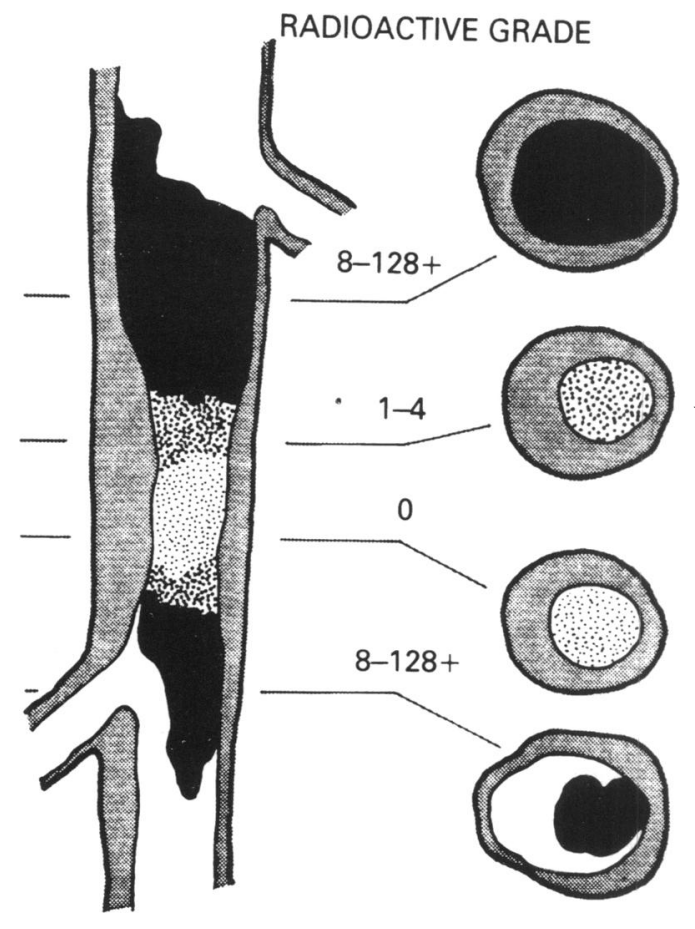

Morphology of acute occlusive lesions

It has long been accepted by pathologists that fatal occlusive thrombosis occurs at the site of severe atherosclerosis ${ }^{12}$ and that recent structural changes in the underlying plaque are usual.

Figure 3 shows the spectrum of morphological types of thrombi found in the 56 cases. Most thrombotic occlusions are complete for only a short axial extent $(2 \mathrm{~mm}$ or less in $19 / 56$ and $2-5 \mathrm{~mm}$ in a further 18 lesions). ${ }^{13}$ The subjacent stenosis is likewise often short. In a few lesions, stenosis, and/or occlusive thrombus may extend for several centimetres.

It is obvious that the prospect of recanalisation by thrombolysis is reduced where the stenosis is long and tortuous and the occlusive thrombus extensive. These features commonly remain undisclosed at pre-treatment clinical angiography; but the extent of the obstruction can be precisely outlined in postmortem injection studies by retrograde filling through anastomoses.

The central portion of the occlusive thrombus is typically pale, from a high content of platelet material and fibrin, and is relatively solid. Characteristically such thrombi were
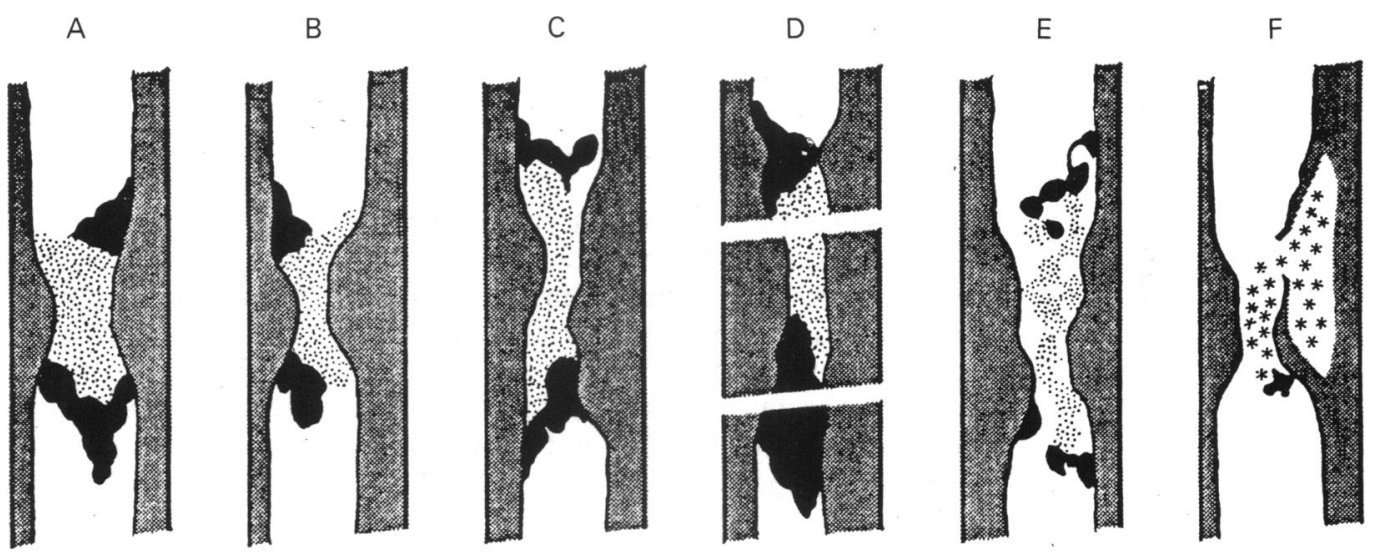

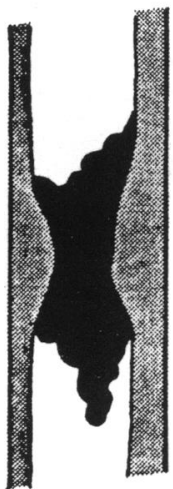

$\mathrm{H}$

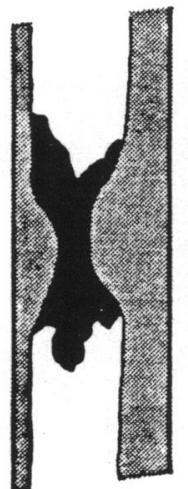

I

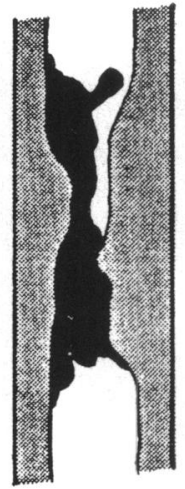

$\mathrm{J}$
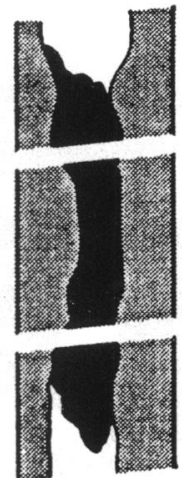

K

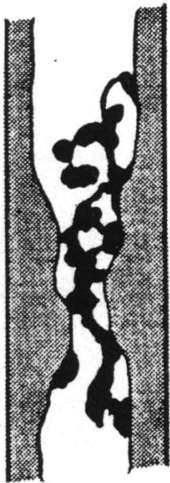

L

Figure 3 Acute coronary occlusion categorised by underlying atherosclerotic narrowing and length, character, and radioactivity of occlusive thrombus (57 cases). Stippling: "cool" thrombus, radioactive grade 0-4. Black: "hot" thrombus, radioactive grade 8-128. (A) Short (1-6 mm) complete occlusion at site of primary thrombus: previous lumen diameter $1 \cdot 3-2 \cdot 0 \mathrm{~mm}$ at narrowest point $(n=16)$. (B) As $A$ but previous lumen diameter $0.5-1 \cdot 2 \mathrm{~mm}$ at narrowest point $(n=19)$. (C) Longer occlusion 6-12 mm approximately; stenosis usually as in $B(n=3)$. (D) Long occlusion $12-60 \mathrm{~mm}$, stenosis as in $A$ or $B(n=4)$. (E) Spongy but highly obstructive thrombus $(n=5)$. (F) Obstruction mainly from atheromatous contents extruded into lumen $(n=1)$. ( $H$ to $L)$ thrombus "hot" throughout $I(n=3), \mathcal{J}(n=2)$, $K(n=2), L(n=2)$. In no instance did thrombus cause complete occlusion where the lumen was greater than $2 \mathrm{~mm}$ in diameter, (about 50\%) at its narrowest point (see Fulton ${ }^{13}$ and Falk ${ }^{15}$ ). Fissure dissection of the plaque underlay thrombosis in most lesions in all categories. 
also radionegative (figs 2 and 3). Propagation of thrombus may then proceed, in the conditions of relative stasis, proximally and/or distally, in either event halting at the next major branch. ${ }^{8}$ Propagated thrombus is usually dark because of its content of red cells, but is not simply whole blood clot. Radiofibrinogen studies showed that it often contained five times as much fibrin as a blood clot. Presumably this reflected pulsatile blood movement in the blind segments of the artery; this would favour access of thrombolytic agents to the face of the thrombus. These studies also showed that propagation proceeded with little delay after primary occlusion-so that every hour, or even minute, could count. Commonly, propagated thrombus only partially occluded the lumen.

Plaques underlying thrombus tend not only to be large but also to differ in some respects from the generality of lesions. Typically, extensive lipid pools are separated from the lumen by a fibrous cap that is thin at least in part.

\section{Proximate causes of thrombosis}

Frequently, but not invariably, plaques subjacent to thrombus are the seat of recent disruptive change (fig 4). This observation is not new. The terminology used, however, has been diverse and includes rents, tears, fissures, rupture, haemorrhage, dissecting haemorrhage, haemorrhagic dissection, dissection, plaque disruption, intimal erosion, and ulceration. ${ }^{6814-17}$ Clearly these changes must usually be antecedent to overlying thrombus and, when present, are generally accepted as its precipitant. Similar changes may be found in other plaques without overlying luminal thrombosis. ${ }^{6818}$

I favour the terms "fissure" for the initial break in the luminal lining and "dissection" (or haemorrhagic dissection) where blood infiltrates the structure of the plaque. These terms describe what actually occurs and reflect somewhat different geneses. Others use "fissure". to encompass both processes. The highest incidences of confirmed fissures related to thrombosis were reported from studies using serial micro-sectioning the full length of the lesion. The results suggest that plaque disruption underlies $70-100 \%$ of occlusive thrombi. ${ }^{14} 1617$

Fissuring typically occurs at points of weakness where the fibrous cap is thin and friable. A fissure may be extensive or minute. ${ }^{16}$ It may lie in the transverse or long axis or obliquely. Elevation of a distal flap may be obstructive. ${ }^{1516}$ Contact with the subendothelial material of the plaque promotes platelet deposition and thrombus formation. This process may merely plug the fissure.

Intimal dissection results when blood forces entry through the fissure into the substance of the plaque. The course taken is governed by the plaque structure:

(a) In relatively fibrous lesions, dissection follows planes of cleavage in the crescentically layered formation of the plaque. ${ }^{81}$ Commonly it works its way towards the outer layers in a short spiral or helical fashion, and may spread proximally and/or distally for several millimetres.

(b) In lesions largely composed of soft amorphous material rich in lipid, dissection can take on an "explosive" character. A mass of blood spreads widely and disruptively, mixing with atheromatous contents, some of which may be extruded into the lumen. Large volume dissections have been described as haematomata. Such lesions may encroach on the lumen ${ }^{1920}$ possibly enough to produce ischaemic symptoms, but complete occlusion from this mechanism alone must be rare.

The fissure tends to occur near the point where stenosis is most severe. Thus in a sample of 43 thrombotic occlusions in which the underlying fissure/dissection could be traced to the lumen, 27 entered at this point, 11 a few $\mathrm{mm}$ proximal, and 5 distal to it. ${ }^{21}$
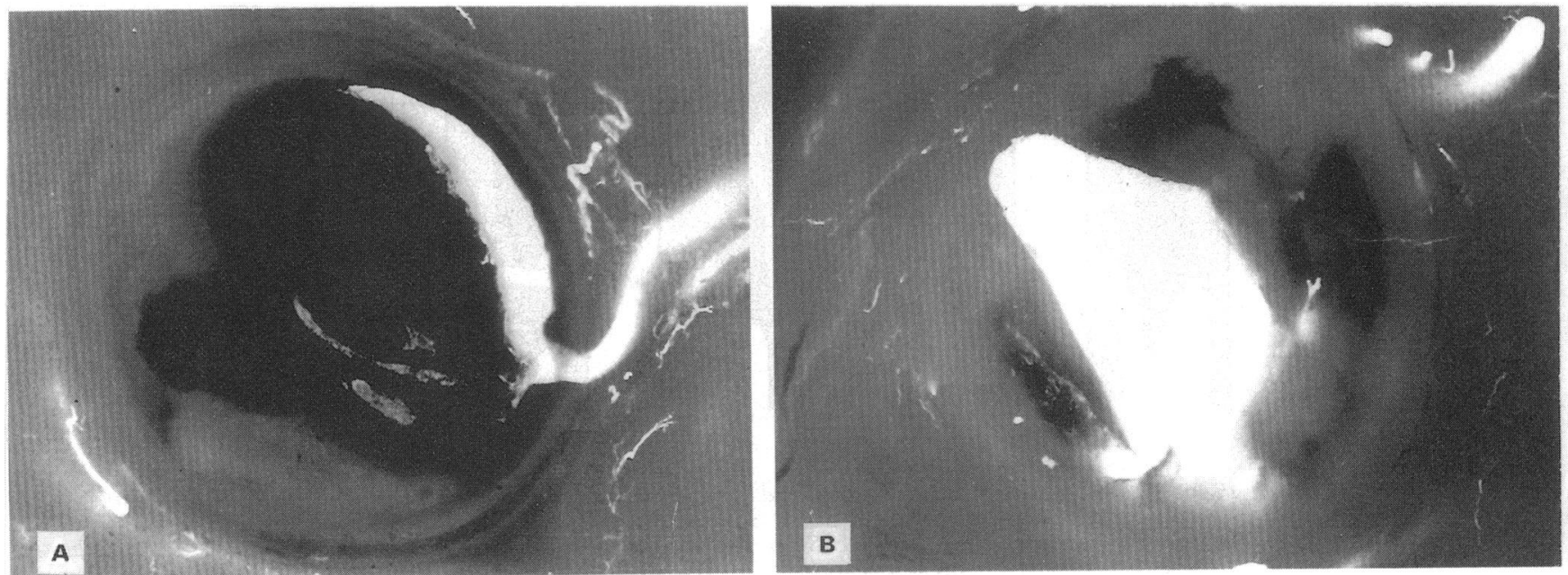

Figure 4 Surface views of $2 \mathrm{~mm}$ thick transverse sections of coronary arteries (unstained $\times 14$ approximately). Thrombus dark and injection medium white (A) Subtotal thrombotic occlusion of lumen, previously about $50 \%$ stenosed. Early "channels" between layers of thrombus are filled by injection white. (A) Subtotal thrombotic occlusion of $o^{\prime}$ 'lock (arrow) with continuity of intraintimal and intraluminal thrombus. (B) Intraplaque thrombus medium. Plaque dissection is present at 8-9 o'clock (arrow) with continuity of intrailuminal thrombus is present. Coincidental finding at necropsy. A small, established intimal vessel arises from the lumen at 3 o'clock. 
It must be emphasised that blood entering the plaque in fissuring and dissection invariably does so from the lumen and the natural process is wholly confined within the original intimal wall, as bounded by the internal elastic lamina. Dissection provoked by catheterisation and angioplasty is another matter.

\section{Intimal haemorrhage}

The term "intimal haemorrhage" is a source of clinical misconception and misgiving, in the context of anticoagulant therapy or thrombolysis. It has been widely used in the past when the preferred description would now be intimal fissuring and dissection. There are good grounds for drawing the distinction:

Haemorrhage implies extravasation of whole blood rich in red cells. The bloodderived material in fissuring and dissection is rich in platelets and fibrin. It is in fact intraintimal thrombus, ${ }^{20}$ not clot. Intraintimal thrombus changes only slowly and, as with unlysed thrombus in the lumen, its fate is to be converted into atherosclerotic material by accumulation of collagen, amorphous substance, and lipid. ${ }^{21}$ It forms thereby a basis for further growth in plaque size and stenosis.

To account for intraintimal thrombus it is necessary to postulate that blood pulses in and out of the fissure/dissection. Intimal thrombus mingles with the atheromatous contents and sometimes thrombus in the lumen also contains atheromatous fragments. ${ }^{6151720}$ Sometimes substantial fragments of atheromatous material are extruded into the lumen and become impacted downstream.

\section{True intimal haemorrhage}

The normal intima is avascular. However, small vessels arising from adventitial arteries (vasa vasorum) do invade the outermost layers of larger atherosclerotic plaques. ${ }^{80-22}$ Not infrequently such vessels give rise to small extravasations of red cells into the intima. ${ }^{80-23}$ There is no secure evidence that such haemorrhages have been precipitants of thrombosis, as has often been assumed.

\section{Other factors responsible for thrombosis over plaques}

Notwithstanding the undeniable pathogenetic association of disruptive changes with coronary thrombosis, the intimal lining seemed to be intact in $3 / 12^{16}$ and $18 / 63^{19}$ occlusions. It must be conceded that even with semitranslucent $2 \mathrm{~mm}$ thick serial sections, minute lesions of the intima escape detection. Many factors may promote platelet aggregation and thrombosis, including microscopic endothelial change, rheological factors in stenosis, and possibly high degrees of coagulability.

Fissuring and dissection are not rare as incidental findings at necropsy (see fig 4). ${ }^{81724}$ So it can be postulated that their occurrence over the span of years contributes a compo- nent of intraintimal thrombus to atherogenesis. ${ }^{82021}$ Thrombosis in the lumen is not an inevitable consequence of fissuring nor is fissuring a prerequisite for thrombosis. But where fissuring/dissection is subjacent to thrombus, as in most instances, it makes sense to accept that the fissuring has led to thrombosis.

Episodic evolution of occlusive thrombus In evolving myocardial infarction the abrupt onset of ischaemic symptoms signals the advent of severe coronary obstruction but not necessarily the moment of complete occlusion. Indeed, layering of thrombus bears witness to its episodic growth ${ }^{132526}$ and was found in about half the lesions. ${ }^{19}$ It is also probable that actual occlusion may occur briefly and intermittently before closure persists $^{151820}$ or alternatively before lasting patency is restored. ${ }^{20}$ These and other coronary and extracoronary features may favourably influence the interval between clinical onset and full commitment to irreversible necrosis-with obvious implications for therapeutic thrombolysis.

\section{Spontaneous recanalisation}

There are few detailed morphological studies of recanalisation of thrombosed coronary arteries. ${ }^{8}$ Successful recanalisation generally begins by separation of thrombus from the arterial wall at one or more sites. Separation may result from shrinkage of the thrombus, relaxation of arterial tonus, and thrombolysis. Early establishment of a channel will favour spontaneous thrombolysis (and also give access to thrombolytic agents). In the course of time a new channel sometimes of useful calibre may form. The residue of unlysed thrombus is incorporated in the artery wall as atherosclerotic material. ${ }^{820}$

Channels may also develop in the substance of the thrombus. Some may persist as intimal vessels, others may link up to effect continuity across the occluding thrombus. A useful restitution of blood flow through such small and often multiple channels is not usual. Narrow tortuous channels commonly become obliterated. ${ }^{8}$

The entire process of canalisation is confined within the previous lumen. ${ }^{827}$ There is no component of vascularisation from adventitial arteries, though small-calibre periarterial anastomoses may sometimes bypass the obstruction.

It seems that early canalisation of thrombotic occlusion not only limits infarct size but favours survival. Accordingly a difference is found between fatal and non-fatal cases. Thus in fatal acute myocardial infarction, occlusion usually remains complete, or nearly so, at death, (44/56 thrombotic occlusions were complete, five were subtotal, and seven spongy, but all were still highly obstructive). By contrast, about half the lesions related to old (therefore survived) infarcts may be patent. Of 116 old obstructive lesions, 81 
were infarct-related and were therefore deemed to have been complete or nearly so at their inception. Of the 81 lesions, 14 were still fully occluded, 29 were traversed by narrow, sometimes multiple channels (hairline to $0.4 \mathrm{~mm}$ in diameter), 27 were severe stenoses $(0.5-1.0 \mathrm{~mm})$, seven were stenoses $(1 \cdot 1-1.5$ $\mathrm{mm}$ ), and four had lumen diameters $>1.5$ $\mathrm{mm} .{ }^{21}$ These findings accord with clinical arteriography performed months or years after infarction. ${ }^{28}$

Clinical coronary arteriography performed within the first day of acute myocardial infarction pointed to early spontaneous recanalisation as a frequent event. Thus occlusion was reported in $89 \%$ of 126 cases of acute transmural infarction examined within 4 hours of the clinical onset but in only $65 \%$ of 57 similar cases at $12-24$ hours of onset. ${ }^{29}$

It is evident that spontaneous recanalisation not only can occur early but indeed must do so if it is to exert a favourable influence on infarct size and survival. It also seems that for the process to be successful morphologically it must not be long delayed. The time course is speculative but the window of opportunity for its initiation may be open only for days. By the time thrombus has undergone autolytic or organisational change, further progress is probably halted and occlusion will persist, indefinitely. ${ }^{8}$

\section{Conclusions}

In the complexities of atherogenesis and coronary artery disease it is evident that thrombus has a wider impact than is generally recognised or acknowledged. That thrombus is the major component in acute coronary occlusion is no longer doubted. Its role in other acute ischaemic states-sudden death, ${ }^{30}$ unstable angina ${ }^{19} 2026$ and acute coronary insufficiency-is becoming more evident.

Coronary artery disease is an episodic process. The atherosclerotic lesions themselves bear witness to the truth of this even in the presymptomatic stages. The final acute arterial occlusion that is signalled by myocardial infarction may be but the latest of a series of episodes involving thrombus. The several acute syndromes of ischaemic heart disease differ not so much in their pathogenetic ingredients as in the rate and severity of their development.

I thank the staff of the Coronary Care Unit and the Departments of Pathology and Radiodiagnosis, Stobhill General Hospital; Dr David J Sumner, Department of Clinical Physics and Bioengineering, West of Scotland Health Boards; and the Department of Health for Scotland for research grants.

1 Fulton WFM. Does coronary thrombosis cause myocardial infarction or vice-versa? In: Weatherall $D$, ed. Advanced medicine. Tunbridge Wells: Pitman Medical,
1978:138-47.

2 Roberts WC, Buja LM. The frequency and significance of coronary arterial thrombi and other observations in fata acute myocardial infarction. Am F Med 1972;52:425-43.

3 Chapman I. The cause-effect relationship between recen coronary artery occlusion and acute myocardial infarction. Am Heart f 1974;87:267-71.

4 Chandler AB. Coronary thrombosis in myocardial infarction. Report of a workshop on the role of coronary thrombosis in the pathogenesis of acute myocardial infarction. Am 7 Cardiol 1974;34:823-33.

5 Davies MJ, Woolf N, Robertson WB. Pathology of acute myocardial infarction with particular reference to occlusive coronary thrombi. Br Heart $\mathcal{F} 1976 ; 38: 659-64$.

6 Ridolfi RL, Hutchins GM. The relationship between coronary lesions and myocardial infarct, ulceration of coronary lesions and myocardial infarct, ulceration of atherosclerotic plaques precipitating
sis. Am Heart $\mathcal{F}$ 1977;93:468-86.

7 Fulton WFM. Chronic generalised myocardial ischaemia with advanced coronary artery disease. $\mathrm{Br}$ Heart $\mathcal{f} 1956$ 18:341-54

8 Fulton WFM. The coronary arteries. Arteriography microanatomy and pathogenesis of obliterative coronary artery disease. Springfield: Charles C Thomas, 1965.

9 Davies MJ, Fulton WFM, Robertson WB. Relation of coronary thrombosis to is

10 Fulton WFM coronary arterial anastomoses and paradoxical changes coronary arterial anastomoses and paradoxical changes
in the subendocardial plexus. Br Heart $\mathcal{f} 1964 ; 26: 39-50$.

11 Fulton WFM. Coronary thrombosis in myocardial infarction. In: Wilhelmsen L, Hjalmarson A, eds. Acute and long-term medical management of myocardia ischaemia. Molndal, Sweden: A Lindren and AB Soner, 1978:59-69.

12 Levine SA, Brown CL. Coronary thrombosis: its various clinical features. Medicine 1929;8:245, 418 .

13 Fulton WFM. Morphology of coronary thrombotic occlusions relevant to thrombolytic intervention. In: Kaltenbach M, Grüntzig A, Rentrop K, Bussmann W-D, eds. Transluminal Coronary Angioplasty and Intracoronary Thrombolysis. Coronary heart disease IV. Berlin: Springer, 1982:244-52.

14 Constantinides P. Plaque fissures in human coronary thrombosis. $₹$ Atheroscl Res 1966;6:1-17.

15 Falk E. Plaque rupture with severe pre-existing stenosis precipitating coronary thrombosis. Characteristics of precipitating coronary thrombosis. Characteristics of coronary atherosclerotic plaque underlying

16 Davies MJ, Thomas AC. The pathological basis and microanatomy of occlusive thrombus formation in human coronary arteries. Philos Trans $R$ Soc Lond [Biol] 1981;294:225-9.

17 Friedman $M$, van den Bovenkamp GJ. The pathogenesis of a coronary thrombus. Am $\mathcal{F}$ Pathol $1966 ; 48: 19-44$

18 Jørgensen $L$, Chandler AB, Borchgrevink CF. Acute lesions of coronary arteries in anticoagulant-treated and in untreated patients. Atherosclerosis 1971;13:21-44.

19 Fulton WFM. Pathogenesis of unstable angina preceding acute myocardial infarction. In: Rafflenbeul W, Lichtlen acute myocardial infarction. In: Rafflenbeul W, Lichtlen Peorge Thiema, 1981:24-30.

20 Davies MJ, Thomas AC. Plaque fissuring: the cause of acute myocardial infarction, sudden ischaemic death and crescendo angina. Br Heart $\mathcal{f}$ 1985;53:363-73.

21 Fulton WFM. Koronaratherosklerose, Fissurbildung de Plaque und Thrombose. In: de Bono D, Brochier $M$ Hugenholtz P, Kübler W, Verstraete $M$, eds. Thrombolytische Therapie des akuten Herzinfarkts. Heidelberg: Springer, 1987:9-40.

22 Winternitz MC, Thomas RM, Le Compte PM. The biology of atherosclerosis. Springfield: Thomas, 1938.

23 Paterson JC. Factors in the production of coronary artery disease. Circulation 1952;6:732-9.

24 Davies MJ, Bland J, Hangartner JB, Angelini A, Thomas AC. Factors influencing the presence or absence of AC. Factors influencing the presence or absence of acute coronary artery thrombi in
death. Eur Heart $f 1989 ; 10: 203-8$.

25 Chapman I. Morphogenesis of occluding coronary artery thrombosis. Arch Pathol 1965;80:256-61.

26 Falk E. Unstable angina with fatal outcome: dynamic coronary thrombosis leading to infarction and/or sudden death. Circulation 1985;71:699-708.

27 Dible JH. Organisation and canalisation in arterial thrombosis. I Pathol Bact 1958;75:1-9.

28 Proudfit WL, Shirey EK, Sones MJ. Selective cine coronary angiography. Correlation with clinical findings in 1000 patients. Circulation 1966;33:901-10.

29 DeWood MA, Spores J, Notske R, et al. Prevalence of total coronary occlusion during the early hours of transtotal coronary occlusion during the early hours of

30 Davies MJ, Thomas AC. Thrombosis and acute coronary artery lesions in sudden cardiac ischaemic death. $N$ Engl artery lesions in sudden ca. 